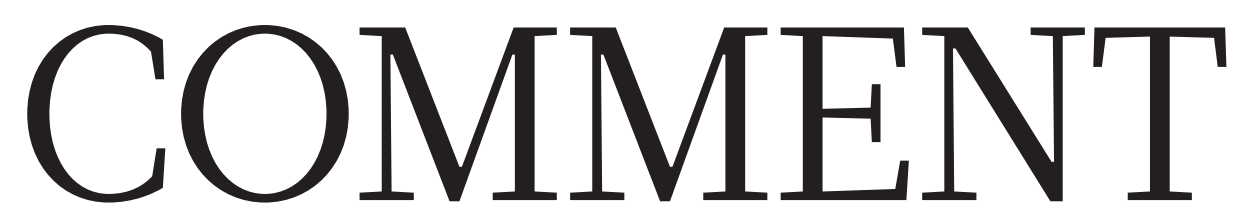

CONSERVATION Four views of the US Endangered Species Act as it turns $40 \mathbf{p . 3 6 9}$

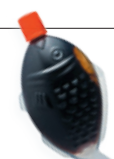

MICROBIoLOgY Harold McGee on festive feasting's debt to fermentation $\mathbf{p . 3 7 2}$
EDUCATION Why Victorian primers taught science by way of fairies p.374
OBITUARY Adrienne Asch, bioethics pioneer, remembered $\mathbf{p . 3 7 7}$

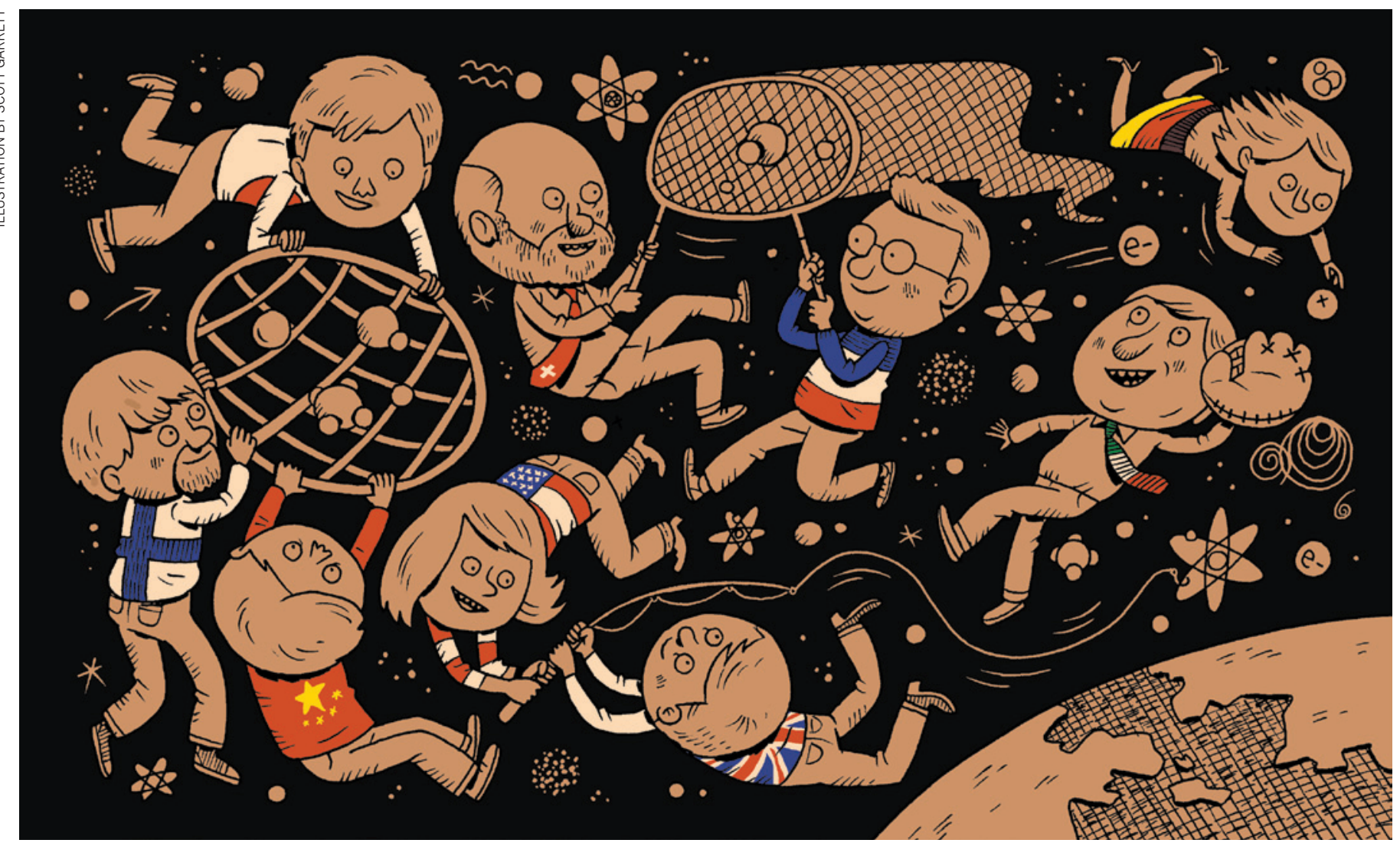

\title{
Together to the next frontier
}

As emerging players jostle old ambitions, Nigel Lockyer calls for the next generation of particle-physics projects to be coordinated on a global scale.

$\mathrm{T}$ his year was a watershed for particle physics. The decades-long quest to discover the Higgs boson is essentially complete. Still abuzz after a Nobel prize for the Higgs prediction, the particle-physics community is feeling satisfied. It is time to pause, reflect and consider what comes next.

The Higgs boson is the last puzzle piece in the standard model of particle physics, but the model does not explain some fundamental aspects of our Universe. From the neutrino's very small mass to dark matter and dark energy, we know there is more going on. But where might the next clue lurk?

We really don't know. Each physicist has his or her own opinion, and countries and regions are preparing to explore different strategies to identify the territories ripest for exploration. What we do know is that the next generation of particle accelerators will be expensive. And requests for government funding will run up against fiscal constraint

$\checkmark$ NATURE.COM See Nature's special issue on the Large Hadron Collider: nature.com/lhc around the globe.

As the incoming director of the Fermi National Accelerator Laboratory (Fermilab) in Batavia,
Illinois, I have spent the past six months in discussions about the future of US particle physics. But particle physics is an international pursuit, with projects in and participants from many different countries. The United States is well positioned to take the lead in some areas, such as neutrino physics, but the global landscape is uncertain. Resources need to be pooled, and new players are emerging. China's and India's talent, infrastructure and ambitions must now be factored into the global equation.

We are at a critical moment for the field. Each country and major project 
$>$ must take into account its impact on the overall enterprise. The opportunity is enormous: a truly coherent approach to accelerate progress and combine disparate strengths. But the risks are just as large: failing to agree on major efforts, losing international partners and sacrificing progress in a downward spiral of too many partially approved projects.

\section{BEYOND THE HIGGS}

The high-energy frontier neighbouring the Higgs will soon be within reach of the Large Hadron Collider (LHC) at CERN, Europe's particle-physics lab near Geneva, Switzerland - currently the highest-energy accelerator in the world. When it turns on again in early 2015 after a two-year upgrade, the machine will operate close to its design energy of 14 teraelectronvolts (TeV) roughly twice that at which the Higgs was discovered. Now that the standard model is complete, any new particles found will revolutionize our view of physics.

Beyond that, there are plans to upgrade the LHC in the 2020s to provide even brighter beams and detectors capable of handling the enormous rates of data that will be produced. Even modest upgrades will cost around US $\$ 1$ billion, requiring contributions from non-member as well as member states.

Other frontiers hold promise. We still do not understand how neutrinos interact, the origin of their tiny masses or their role in the early Universe. Fermilab is heading a US proposal to build a long neutrino-beam experiment, running 1,300 kilometres from Fermilab to the Homestake mine in South Dakota. An ambitious 35-kilotonne liquid argon detector located nearly 1,500 metres below the surface emerged as the preferred project when the US community met in Minnesota for a ten-day planning symposium in July. It would help us to understand neutrino masses and whether these particles contribute to the matter-antimatter asymmetry of the Universe.

With the total construction budget nearing $\$ 1$ billion, the experiment will require international partners - a new approach for US domestic science. The US Department of Energy's Office of Science has indicated that it would support such a major proposal if there was involvement from Europe and Asia.

A long-baseline neutrino experiment is necessary, somewhere. Alternatives are on the table: one in Europe would see neutrinos travelling from CERN to Finland; another has neutrinos travelling across Japan. But the world can afford only one.

Japan is perhaps the stiffest competitor, with leading programmes in neutrino physics, a bottom-quark ('b-quark') factory and kaon and muon experiments. The country hopes to host the International Linear Collider (ILC) - a 30-kilometre-long, 500-gigaelectronvolt electron-positron collider to study the Higgs boson that would surpass the LHC in terms of precision in the late 2020s. It is hoped that construction will begin later this decade.

Japan is about to begin a campaign to sign up support for this international project, which would require US and European participation. Most particle physicists support the ILC but many would like to see what discoveries will come out of the upgraded LHC first. If no new particles emerge, then the higher precision available to the ILC would make building it even more attractive. If another discovery is made at the LHC, then the community might need a different machine to explore the new energy regime.

\section{PASSING THE BATON}

Given that we are not sure which is the next fertile energy range, many particle physicists believe that we should aim as high as possible. Some argue for an even higher-energy lepton collider, such as a muon collider or the Compact Linear Collider (a European higher-energy competitor to the ILC) that would reach $3-5 \mathrm{TeV}$. Europe is assembling a team to design a $100-\mathrm{TeV}$ proton-proton collider, with a 100-kilometre-circumference tunnel, to probe any particles that may be discovered by the upgraded LHC and at energies beyond. This machine could start up in the 2030s.

And the United States still has ambitions to host a high-energy frontier machine, after turning off the "If China does Fermilab's Tevatron jump ahead, accelerator in 2011 it will change and failing to realize the landscape the Superconducting of science." Super Collider in the 1990s. Perhaps the high-energy baton could be passed back to the United States. Fermilab is still a world leader in high-field magnets for proton accelerators, which would be necessary for any $100-\mathrm{TeV}$ proton-proton collider.

To add to the suspense, there is the changing role of China. Historically a small player in particle physics, it last year stepped onto the world stage with impressive results in reactor-driven neutrino physics, including showing that two of the three types of neutrinos mix together much more than expected. This large mixing implies that fundamental symmetry differences between neutrinos and antineutrinos might be observable in a long-baseline experiment, telling us about matter and antimatter imbalances in the early Universe. Emboldened, might the Chinese leapfrog the world by hosting the $100-\mathrm{TeV}$ machine? Construction of the machine would be cheaper in China, although the nation would need help from the rest of the world to design and build it.

If China does jump ahead, it will change the landscape of science, levelling the playing field with the emerging economies. Discussions about global progress in particle physics would need to explicitly include heads of state from China and India as well as North America, Europe and Japan.

\section{GLOBAL CONVERSATION}

Physicists do not care where they do their research. But the vast scale of particlephysics projects means that all new megaaccelerators require global planning, agreement and joint construction. Governments around the world will have to make unprecedented financial investments in other countries, challenging the traditional political calculus in which public tax dollars are primarily spent at home for direct, short-term benefit.

Governments are trying to understand which models best suit their national interests. There is less talk of 'brain gain and drain' and more of 'brain circulation'. Countries and intellectual communities thrive by participating in the global conversation, not necessarily by owning most of the players.

Care must be taken to ensure the health of major regional laboratories, such as CERN, Fermilab and the High Energy Accelerator Research Organization (KEK) in Tsukuba, Japan: these are the only places where large particle-physics projects are currently feasible. Demands from emerging economies such as China to host other projects will challenge the long-term plans of the existing leaders. Scientists in the United States and Europe will have to find out how best to use international competition as a spur for advancing projects on their own soil while still being good international partners. This may become tricky.

Higgs bosons are not export-controlled, nor are pictures of deep space from advanced telescopes. But the technologies developed, often through international collaborations, may have dual use - for defence applications or for economic gains, for example, as well as for basic science. Countries will have to decide how to oversee and exploit these opportunities responsibly.

Particle-physics leaders need to be more vocal and aggressive in defining and defending the global programme. After all, a suite of global particle-physics facilities helps us to understand how the Universe works.

Nigel Lockyer is director of the Fermi National Accelerator Laboratory in Batavia, Illinois.

e-mail:lockyer@fnal.gov 